\title{
Identification of multiple risk variants for ankylosing spondylitis through high-density genotyping of immune-related loci
}

\author{
International Genetics of Ankylosing Spondylitis Consortium (IGAS) ${ }^{*}$
}

\begin{abstract}
Ankylosing spondylitis is a common, highly heritable inflammatory arthritis affecting primarily the spine and pelvis. In addition to $H L A-B^{*} 27$ alleles, 12 loci have previously been identified that are associated with ankylosing spondylitis in populations of European ancestry, and 2 associated loci have been identified in Asians. In this study, we used the Illumina Immunochip microarray to perform a case-control association study involving 10,619 individuals with ankylosing spondylitis (cases) and 15,145 controls. We identified 13 new risk loci and 12 additional ankylosing spondylitis-associated haplotypes at 11 loci. Two ankylosing spondylitis-associated regions have now been identified encoding four aminopeptidases that are involved in peptide processing before major histocompatibility complex (MHC) class I presentation. Protective variants at two of these loci are associated both with reduced aminopeptidase function and with MHC class I cell surface expression.
\end{abstract}

Inflammatory arthritis in ankylosing spondylitis causes pain and stiffness and progressively leads to new bone formation and ankylosis (fusion) of affected joints. It affects $0.55 \%$ of populations of European ancestry (herein termed Europeans) ${ }^{1}$ and $0.23 \%$ of Chinese ${ }^{2}$, but is uncommon in Africans and Japanese, mostly owing to the low prevalence in these ancestry groups of $H L A-B^{\star} 27$, the major genetic variant associated with ankylosing spondylitis. Whereas effective treatments are available that suppress inflammation and improve symptoms, there are not yet any treatments that have been shown to robustly slow the rate of ankylosis or induce disease remission.

Ankylosing spondylitis is highly familial (sibling recurrence risk ratio of $>52)^{3}$ and heritable $\left(h^{2}>90 \%\right)^{4}$. It is two to three times more prevalent in men than in women, and men tend to be more severely affected. More than $80 \%$ of cases are positive for the $H L A-B^{\star} 27$ allele, but only a minority of $H L A-B^{\star} 27$ carriers develop ankylosing spondylitis (1-5\%). The low proportion of $H L A-B^{\star} 27$ carriers who develop ankylosing spondylitis reflects the fact that numerous other non-HLA- $B^{\star} 27$ variants are likely to influence disease susceptibility ${ }^{3}$. In addition to $H L A-B^{\star} 27,12$ loci have previously been confirmed to be associated with ankylosing spondylitis in Europeans (ANTXR2, CARD9, ERAP1, IL12B, IL23R, KIF21B, PTGER4, RUNX3, TBKBP1, TNFRSF1A and chromosomes $2 \mathrm{p} 15$ and $21 \mathrm{q} 22)^{5-7}$, and 2 loci have recently been reported in Han Chinese (HAPLN1-EDIL3 and ANO6) ${ }^{8}$.

The Immunochip Consortium has developed a custom microarray SNP genotyping chip (the Immunochip), the design of which has been informed by available genome-wide association study (GWAS) and deep sequencing data from various autoimmune and inflammatory diseases to provide a cost-effective platform for immunogenetic studies $^{9,10}$. Genetic data from ankylosing spondylitis, psoriasis,
Crohn's disease and ulcerative colitis, along with several classic autoimmune diseases, were used in the chip design, making it a powerful platform for studies of pleiotropic genetic effects in these related diseases. In this study, we aimed to identify new associations with ankylosing spondylitis and to dissect and refine the boundaries of known associated loci by performing a dense SNP genotyping study in 10,619 cases and 15,145 controls of European, East Asian and Latin American ancestry using the Immunochip.

\section{RESULTS}

Primary association findings

After all sample quality control filters, the European cohort consisted of 9,069 cases and 13,578 controls, and the East Asian cohort consisted of 1,550 cases and 1,567 controls. The genomic inflation factor $(\lambda)$ calculated using 1,922 SNPs included on the Immunochip from studies of reading and writing ability, psychosis and schizophrenia was $1.047\left(\lambda_{1000}\right.$ for an equivalent study of 1,000 cases and 1,000 controls $=1.0285)$, indicating minimal evidence of residual population stratification in the overall data set (quantile-quantile plots are presented in Supplementary Fig. 1).

Association at genome-wide significance $\left(P<5 \times 10^{-8}\right)$ was observed for 25 loci, including the MHC (Table 1 and Supplementary Fig. 2; genomic control-corrected results are shown in Supplementary Table 1). Suggestive association $\left(P<5 \times 10^{-7}\right)$ was observed at six additional loci (Supplementary Table 2). As with all GWAS, there is uncertainty as to the genes contributing to association at specific loci. At previously reported loci, association $\left(P<5 \times 10^{-8}\right)$ was seen with the most strongly associated previously reported SNPs at CARD9, ERAP1, IL12B, IL23R, KIF21B, RUNX3, NPEPPS-TBKBP1-TBX21,

${ }^{*}$ A list of authors and affiliations appears at the end of the paper. 


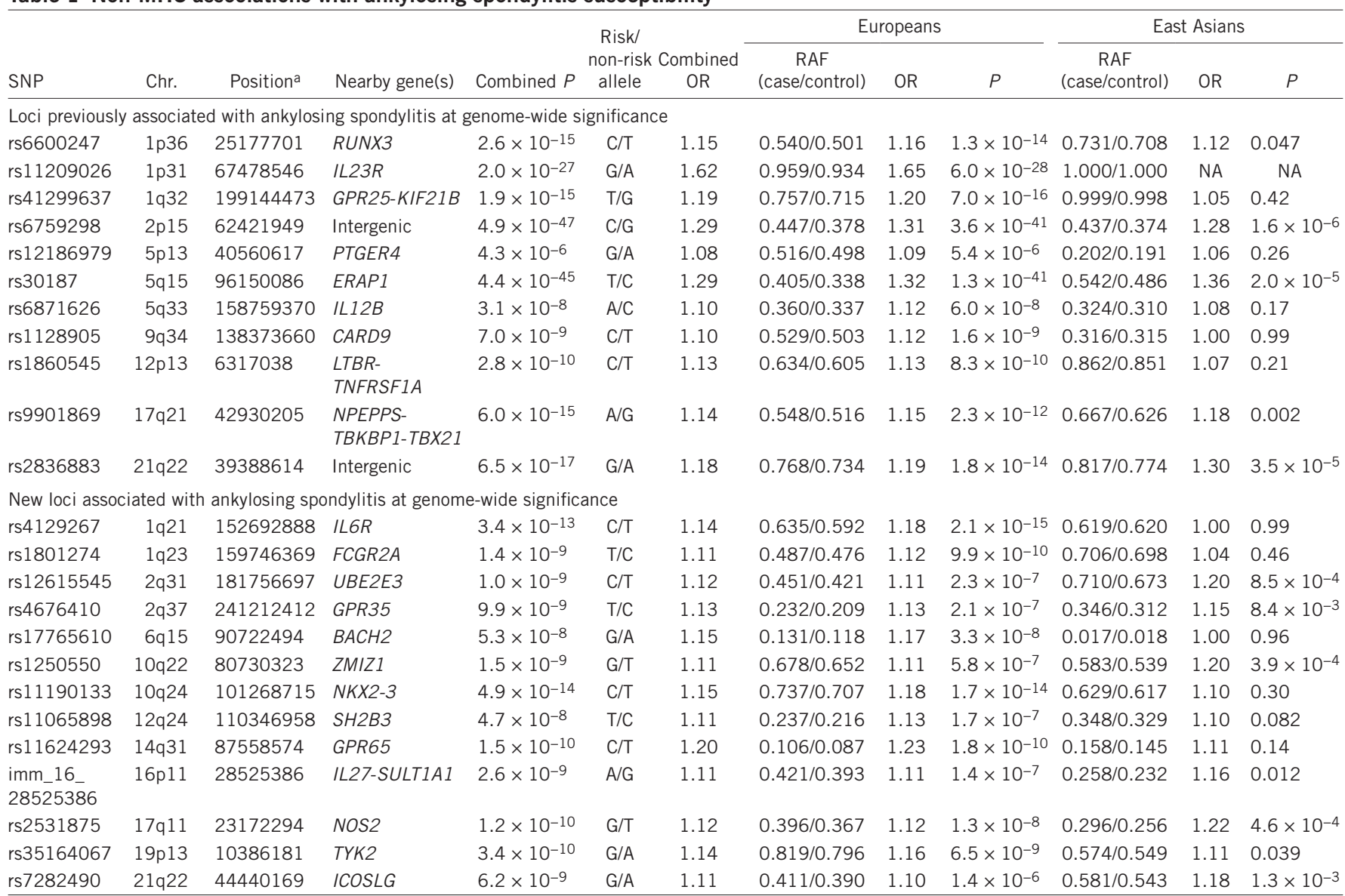

Locus plots for reported associations are shown in Supplementary Figure 5. Chr., chromosome; RAF, risk allele frequency; NA, not available.

aNCBI Build 36 human genome coordinates.

TNFRSF1A and chromosomes 2p15 and 21q22 (Table 1). At PTGER4, the previously associated SNP (rs10440635) also showed moderate association in the current study $\left(P=3.0 \times 10^{-5}\right.$; imputed $)$. No SNPs at the ANTXR2 locus were included on the Immunochip.

We observed little evidence of association with the two previously reported loci in Han Chinese, either in Europeans, East Asians (Chinese, Taiwanese and Koreans) or in the combined data set $(P>0.05)^{8}$. To increase power for these variants, we genotyped a total of 2,998 East Asian cases and 5,547 East Asian controls. No association was seen $(P>0.05)$ for either rs4552569 (chromosome 5q14, between HAPLN1 and EDIL3) or rs17095830 (chromosome 12q12, ANO6) (Supplementary Table 3). rs4552569 showed only nominal significance in Europeans $(P=0.02)$. rs17095830 was not directly typed on the Immunochip, but, in a previous $\mathrm{GWAS}^{6}$, no association was observed with this SNP $(P>0.1)$.

Genome-wide significance was seen at 13 loci not previously known to be associated with ankylosing spondylitis (Table 1). The strongest association at each locus was with a common variant (minor allele frequency $(\mathrm{MAF})>5 \%)$, but several associations were also seen with rare variants $(\mathrm{MAF}<1 \%)$ at these loci, including in the genes CARD9, IL23R, LNPEP and TYK2. Both rare variants in $I L 23 R$ were nonsynonymous coding variants, whereas the CARD9, TYK2 and LNPEP variants were located at exon-intron boundaries and were predicted to influence splicing.

In total, $24.4 \%$ of the heritability of ankylosing spondylitis is now explained: $4.3 \%$ from loci other than $H L A-B$ and $20.1 \%$ due to $H L A-B^{\star} 27$ itself.

\section{IL-23 pathway genes}

Genetic studies provided the first evidence that interleukin (IL)-23 is involved in the pathogenesis of ankylosing spondylitis, and variants in several genes involved in the IL-23 proinflammatory cytokine pathway have been shown to be associated with the disease. This study adds to that list, with loci containing TYK2, IL6R and IL27 achieving genome-wide significance.

IL-6 signaling through IL-6R has diverse proinflammatory effects. rs4129267, the most strongly associated IL6R SNP in this study, is also associated with asthma ${ }^{11}$ but with the opposite direction of association to ankylosing spondylitis. The allele associated with risk of ankylosing spondylitis at this SNP is strongly associated with lower serum concentrations of the soluble form of the IL-6 receptor (sIL-6R), with each allele associated with a 1.4 -fold variation in serum IL-6R concnetrations ${ }^{12}$. We found that sIL-6R concentrations varied strongly by rs4129267 genotype, both overall and separately, in cases and controls (Fig. 1). Overall, homozygous carriers of the T allele at rs 4129267 had sIL-6R concentrations $73 \%$ higher than homozygous carriers of the C allele ( 28.9 versus $\left.16.7 \mathrm{ng} / \mathrm{ml} ; P=7.8 \times 10^{-17}\right)$. This SNP has previously been associated with serum $C$-reactive protein (CRP) concentrations at genome-wide significance; in the current study, serum CRP concentrations were $30 \%$ higher in cases homozygous for the T allele than in cases homozygous for the C allele (19.2 versus $14.8 \mathrm{mg} / \mathrm{l}$ ).

At $I L 23 R$, we previously identified two independent diseaseassociated haplotypes ${ }^{6}$ tagged by rs 11209026 and rs11209032. Here, after conditioning on rs11209026, the strongest association was with 


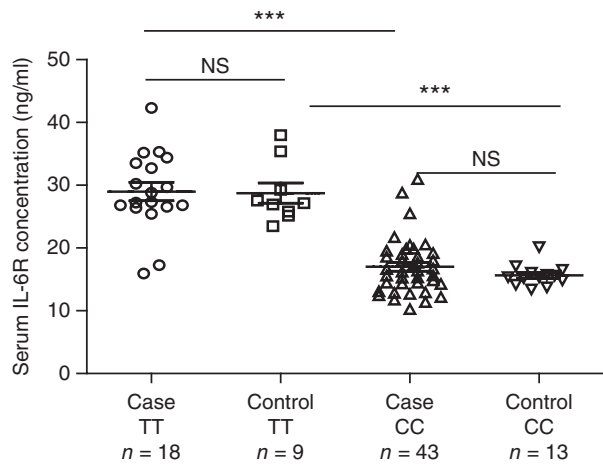

Figure 1 IL6R polymorphism alters IL-6R serum concentrations. IL-6R concentrations were determined in cases and controls who were homozygous for either the T or C allele at the rs4129267 SNP. In both cases and controls, individuals homozygous for the $\mathrm{C}$ allele showed significantly lower concentrations of circulating IL-6R. ${ }^{* * *} P<0.0001$; NS, not significant. Bars represent mean \pm s.e.m.

rs 12141575 (odds ratio $\left.(\mathrm{OR})=1.15 ; P=9.4 \times 10^{-11}\right)$; this SNP is in strong linkage disequilibrium (LD) with rs11209032 $\left(r^{2}=0.993\right)$ and also with rs1495965, which has been reported to be associated with Behçet's disease $\mathrm{e}^{13,14}$. Behçet's disease is complicated by sacroiliitis resembling ankylosing spondylitis in up to $10 \%$ of cases ${ }^{15}$.

Six loci showed suggestive association $\left(5 \times 10^{-8}<P<5 \times 10^{-7}\right)$, including a region on chromosome 2 q11 encoding IL1R2 and ILIR1. Conditional analyses showed that there were two separate signals at this locus (Table 2), one in each gene. Both genes encode receptors for the cytokine IL-1, which, among diverse proinflammatory functions, also promotes $\mathrm{T}$ helper $17\left(\mathrm{~T}_{\mathrm{H}} 17\right)$ lymphocyte differentiation.

\section{Aminopeptidase genes}

We previously identified a strong association between ERAP1 and ankylosing spondylitis ${ }^{5}$ that is restricted to $H L A-B^{\star} 27$ positive disease ${ }^{6}$. This observation was replicated here (Table 3 and Supplementary Figs. 3 and 4), with strong interaction observed in the European cases between $H L A-B^{\star} 27$ and both independently associated ERAP1 haplotype-tagging SNPs (rs30187: $\beta=0.390$, $P=2.9 \times 10^{-11}$; rs 10045403: $\beta=-0.282, P=9.3 \times 10^{-6}$; Supplementary Table 4). We observed no evidence of epistasis between $H L A-B^{\star} 27$ and rs30187 (or other SNPs in ERAP1) in the East Asian samples, probably because of inadequate statistical power. In this analysis, we had $35 \%$ power to detect an association at a significance level of $P<0.05$ with the observed allele frequency of rs30187 in controls and the effect size observed in the overall analysis. The low power to detect a major effect at rs30187 in $H L A-B^{\star} 27$-positive East Asian samples suggests that the power to detect an interaction would be quite small. The observed OR $\left(95 \%\right.$ confidence interval (CI)) in $H L A-B^{\star} 27$-positive subjects at rs30187 was $0.73(0.53-1.03)$ compared to $1.20(0.89-1.61)$ in $H L A-B^{\star} 27$-negative subjects. Although these analyses did not detect significant interaction $(P>0.05)$, they are consistent with an interaction between rs30187 and $H L A-B^{\star} 27$, where rs30187 is only associated in $H L A-B^{\star} 27$-positive disease. This finding warrants further exploration with larger sample sizes in East Asian populations.

LNPEP and ERAP2 are both members of the endoplasmic reticulum (ER) aminopeptidase family and have substantial sequence homology with ERAP1. They are both encoded on chromosome 5q15, immediately centromeric to the ERAP1 locus. A previous study, which did not control for the association of ERAP1 with ankylosing spondylitis, observed no association of an ERAP2 loss-of-function variant with ankylosing spondylitis ${ }^{16}$. Other studies have identified associations with ERAP2 but have not dissected them from the known associations of ERAP1 SNPs ${ }^{5,17}$. Here, controlling for the association of ERAP1 with ankylosing spondylitis, two functionally important SNPs in ERAP2 were found to be associated with ankylosing spondylitis: rs2549782, which leads to a change in ERAP2 catalytic activity ${ }^{18}$, and rs2248374, where the protective $\mathrm{G}$ allele causes complete loss of ERAP2 mRNA and absence of ERAP2 protein ${ }^{19}$. In the European samples, controlling for the association with ERAP1, we identified SNPs in ERAP2 and $L N P E P$ that were associated with ankylosing spondylitis (lead SNP, rs2910686: OR $=1.2, P=4.5 \times 10^{-17}$; Supplementary Fig. 3 b). Because the association of ERAP1 variants was restricted to HLA$B^{\star} 27$-positive ankylosing spondylitis, analyzing ERAP2 and LNPEP SNPs in $H L A-B^{\star} 27$-negative cases and controls produced similar results to analyses of the combined (HLA-B $B^{\star} 27$-positive and $H L A-B^{\star} 27$-negative) cases and controls when the association with ERAP1 was controlled for. Thus, we observed association with ERAP2 SNPs in $H L A-B^{\star} 27-$ negative ankylosing spondylitis cases (rs2910686: $\left.\mathrm{OR}=1.19, P=2.13 \times 10^{-5}\right)$. To investigate the possibility that this finding might be an artifact caused by the strong LD between the ERAP2 locus and $H L A-B^{\star} 27$ in cases, we tested association at this locus in a multivariate analysis, controlling in the one analysis for association of the two ankylosing spondylitis-associated ERAP1 haplotypes (tagged by rs30187 and rs10045403), their interaction with $H L A-B^{\star} 27$ and $H L A-B^{\star} 27$ itself. In this analysis, association with rs2910686 was robust $\left(P=6.6 \times 10^{-8}\right)$, suggesting that $E R A P 2-L N P E P$ is independently associated with ankylosing spondylitis. Haplotype counts for rs 2910686 and rs30187 in both $H L A-B{ }^{*} 27$-positive and $H L A-B^{\star} 27$-negative cases are shown in Table 3.

Table 2 Secondary signals in Europeans at loci known to be associated with ankylosing spondylitis

\begin{tabular}{|c|c|c|c|c|c|c|c|c|c|}
\hline Chr. & SNP & Position & Nearby gene(s) & $\begin{array}{c}\text { Risk/non-risk } \\
\text { allele }\end{array}$ & Conditional SNP & $P$ & OR & $\begin{array}{c}\text { RAF } \\
\text { (case/control) }\end{array}$ & $\begin{array}{l}\mathrm{LD}\left(r^{2} / D^{\prime}\right) \text { with } \\
\text { conditional SNP }\end{array}$ \\
\hline $1 \mathrm{p} 31$ & rs12141575 & 67520024 & IL23R & $A / G$ & rs11209026 & $9.4 \times 10^{-11}$ & 1.15 & $0.370 / 0.330$ & $0.034 / 0.983$ \\
\hline $1 q 23$ & rs2039415 & 159121069 & FCGR2A & $\mathrm{C} / \mathrm{T}$ & rs1801274 & $7.4 \times 10^{-5}$ & 1.09 & $0.702 / 0.682$ & $0.002 / 0.062$ \\
\hline $2 q 12$ & rs2192752 & 102135805 & ILIR2-ILIR1 & $\mathrm{C} / \mathrm{A}$ & rs4851529 & $4.1 \times 10^{-6}$ & 1.11 & $0.239 / 0.222$ & $0.007 / 0.192$ \\
\hline $5 q 15$ & rs10045403 & 96173489 & ERAP1-ERAP2 & $A / G$ & rs30187 and rs2910686 & $5.8 \times 10^{-14}$ & 1.20 & $0.783 / 0.730$ & $\begin{array}{l}0.178 / 0.958 \\
0.091 / 0.429\end{array}$ \\
\hline $5 q 15$ & rs2910686 & 96278345 & ERAP1-ERAP2 & $\mathrm{C} / \mathrm{T}$ & rs30187 & $4.5 \times 10^{-17}$ & 1.17 & $0.450 / 0.440$ & $0.153 / 0.617$ \\
\hline $5 q 33$ & rs6556416 & 158751323 & $I L 12 B$ & $\mathrm{C} / \mathrm{A}$ & rs6871626 & $4.4 \times 10^{-6}$ & 1.11 & $0.704 / 0.675$ & $0.048 / 0.443$ \\
\hline $6 q 15$ & rs639575 & 91047852 & $\mathrm{BACH} 2$ & $\mathrm{~A} / \mathrm{T}$ & rs17765610 & $8.6 \times 10^{-5}$ & 1.08 & $0.624 / 0.609$ & $0.000 / 0.042$ \\
\hline $12 \mathrm{p} 13$ & rs7954567 & 6361386 & LTBR-TNFRSFIA & $A / G$ & rs1860545 & $1.2 \times 10^{-7}$ & 1.11 & $0.363 / 0.341$ & $0.002 / 0.068$ \\
\hline $16 p 11$ & rs35448675 & 28236248 & IL27-SULT1A1 & $A / G$ & imm_16_28525386 & $2.4 \times 10^{-4}$ & 1.24 & $0.007 / 0.006$ & $0.003 / 0.955$ \\
\hline $17 q 11$ & rs2297518 & 23120724 & Nos2 & $A / G$ & rs2531875 & $6.3 \times 10^{-7}$ & 1.13 & $0.212 / 0.190$ & $0.000 / 0.048$ \\
\hline $19 p 13$ & rs6511701 & 10486067 & TYK2 & $\mathrm{A} / \mathrm{C}$ & rs35164067 & $1.4 \times 10^{-4}$ & 1.10 & $0.220 / 0.218$ & $0.162 / 0.419$ \\
\hline
\end{tabular}


Table 3 Association analysis of rs30187 and rs2910686 haplotypes in samples positive and negative for $H L A-B * 27$

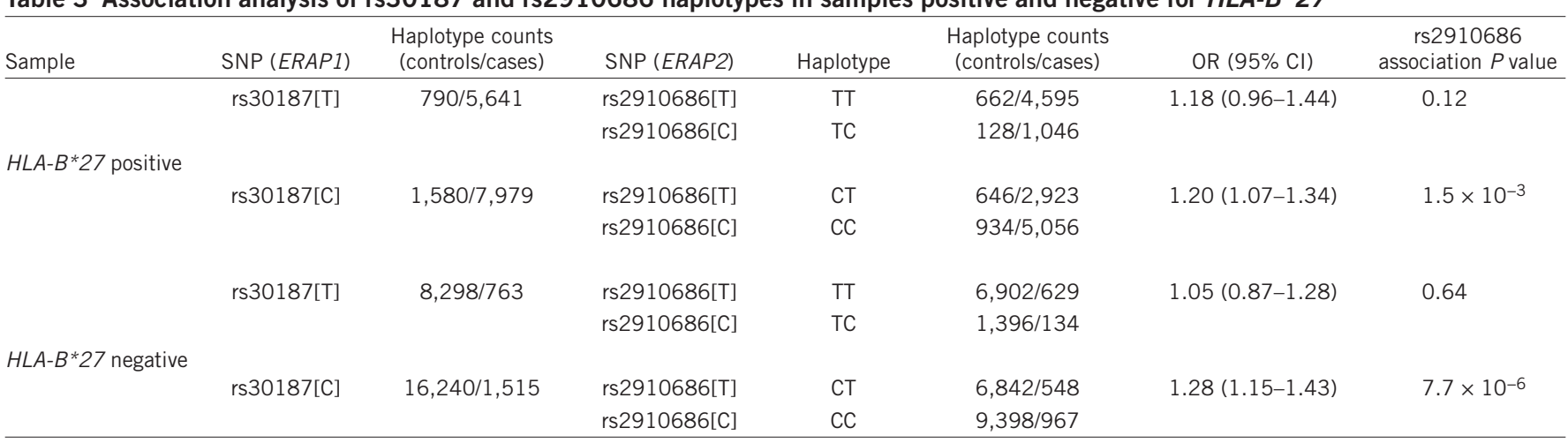

Association was assessed by 1-degree-of-freedom $\chi^{2}$ test.

In addition to these ERAP1 and ERAP2 associations, we observed genome-wide significant association of SNPs on chromosome 17q21 around the gene NPEPPS, which encodes puromycin-sensitive aminopeptidase (rs9901869: OR $=1.14, P=6.0 \times 10^{-15}$ ) (Supplementary Fig. 5). The NPEPPS protein localizes to the cytoplasm and is thought to be involved in processing proteasome-derived peptides before their transport to the endoplasmic reticulum and presentation by human leukocyte antigen (HLA) class I molecules ${ }^{20}$. Association had previously been reported at this locus and was ascribed to TBKBP1 or TBX21. Here SNPs mapping to NPEPPS and TBX21 were independently associated with ankylosing spondylitis, and conditional analysis suggested that there are at least two independent signals at this locus. When conditioning on rs9901869, SNP rs11657479 in the 3' UTR of TBX21 remained significantly associated with ankylosing spondylitis $\left(\mathrm{OR}=1.09 ; P=1.8 \times 10^{-3}\right)$. The data did not allow us to determine whether TBKBP1 or TBX21 was primarily associated with ankylosing spondylitis, but both represent attractive candidates. TBKBP1 is a component of the tumor necrosis factor (TNF) signaling pathway, and TBX21 is a transcription factor that influences the differentiation of $\mathrm{T}$ helper $1\left(\mathrm{~T}_{\mathrm{H}} 1\right)$ and natural killer $(\mathrm{NK})$ cells $^{21}$.

\section{Genes influencing lymphocyte activation and differentiation}

It has recently been shown that cell type-specific trimethylation of histone $\mathrm{H} 3$ at lysine $4(\mathrm{H} 3 \mathrm{~K} 4 \mathrm{me} 3)$ chromatin marks can inform the fine mapping of associated SNPs to identify causal variation ${ }^{22}$. We therefore tested all ankylosing spondylitis-associated SNPs against H3K4me3 chromatin marks in different cell lines from the Encyclopedia of DNA Elements (ENCODE) Project ${ }^{23}$. This analysis showed a strong enrichment of disease-associated SNPs associated with H3K4me3 chromatin marks in cells of immune origin (Supplementary Fig. 6).

Because of this association and taking into account the likely pathogenic role of T lymphocytes in ankylosing spondylitis and the involvement of several genes associated with ankylosing spondylitis in T-lymphocyte differentiation, we tested association of the SNPs for ankylosing spondylitis with $\mathrm{CD}^{+}$and $\mathrm{CD} 8^{+} \mathrm{T}$ cell counts in a previously published GWAS data $\operatorname{set}^{24}$. Association $(P<0.005)$ was seen between $\mathrm{CD}^{+}$lymphocyte counts and ankylosing spondylitisassociated SNPs in the loci harboring the genes $I L 7 R, R U N X 3$ and ZMIZ1 (Supplementary Table 5). Association was also observed between SNPs in EOMES and CD8 ${ }^{+}$lymphocyte counts, but these were not the same EOMES SNPs that were associated with ankylosing spondylitis. We also showed association of the genes $\mathrm{SH} 2 \mathrm{~B} 3$ and $B A C H 2$ with both ankylosing spondylitis and $\mathrm{CD}^{+}$lymphocyte counts (Supplementary Table 5). We previously showed that CD8 ${ }^{+}$ lymphocyte counts are lower in ankylosing spondylitis cases than in healthy age- and sex-matched controls ${ }^{6}$. In contrast, in this study, we found that ankylosing spondylitis cases not on biological therapy had similar $\mathrm{CD}^{+}$lymphocyte counts as age-matched controls (Supplementary Fig. 7).

\section{HLA Region}

After SNP imputation in the MHC region, rs116488202 was found to tag $H L A-B^{\star} 27$ more accurately in both Europeans and Asians than our previously reported tagging SNP rs4349859 and also rs13202464, reported to tag $H L A-B^{\star} 27$ in Asian populations ${ }^{8}$ (Supplementary Table 6). The expected strong association was observed with $H L A-B^{\star} 27\left(\mathrm{OR}=46 ; P<1 \times 10^{-100}\right)$ (Supplementary Fig. 8). Risk of ankylosing spondylitis was further increased in $H L A-B^{\star} 27$ homozygotes; $H L A-B^{\star} 27$ homozygosity was more prevalent in $H L A-B^{\star} 27$-positive cases than in $H L A-B^{\star} 27$-positive controls $(\mathrm{OR}=2.07 ; P=0.0025)$.

Controlling for association with $H L A-B^{\star} 27$, there was residual signal with SNPs near $H L A-A$ and $H L A-B$ (Fig. 2). The residual signal at $H L A-B$ may reflect either imperfect $H L A-B^{\star} 27$ tagging by rs116488202 or association of other $H L A-B$ alleles with ankylosing spondylitis. No other individual non $-H L A-B^{\star} 27$ allele was associated with ankylosing spondylitis, although this may represent imperfect $H L A-B$ imputation using single SNPs.

The most strongly associated SNP near $H L A-A$, rs2394250, tags the classical allele $H L A-A^{\star} 0201$ (Supplementary Table 7). Association of the $H L A-A^{\star} 0201$ allele was independent of $H L A-B^{\star} 27$ genotype, present in both $H L A-B^{\star} 27$-positive $\left(\mathrm{OR}=1.21, P=6.5 \times 10^{-12}\right.$; conditioning on rs116488202) and $H L A-B^{\star} 27-$ negative $(\mathrm{OR}=1.36$, $P=3.2 \times 10^{-13}$ ) disease (Supplementary Table $7 \mathbf{b}$ ). No significant correlation was noted between $H L A-B^{\star} 27$ and SNPs tagging

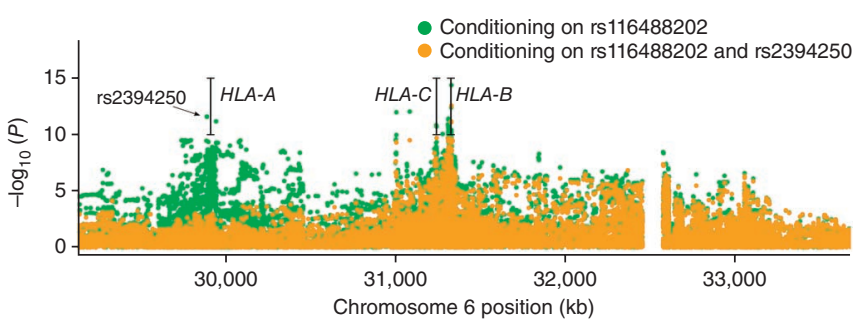

Figure 2 Ankylosing spondylitis susceptibility associations in the $\mathrm{MHC}$ region conditioning on the $H L A-B * 27$-tagging SNP rs 116488202 and further conditioning on the $H L A-A{ }^{*} 02$-tagging SNP rs2394250.

The 85-kb gap between positions $32,465 \mathrm{~kb}$ and $32,550 \mathrm{~kb}$ corresponds to an assembly correction between NCBI Genome Builds 36 and 37 of the human genome. 


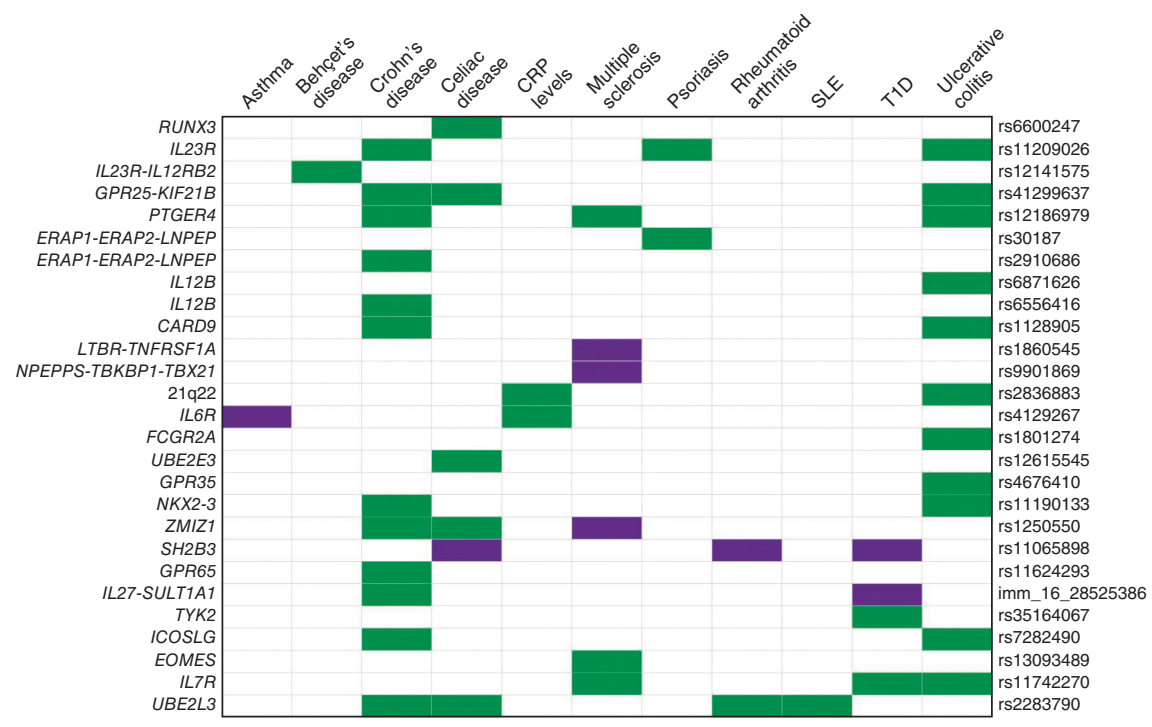

Figure 3 Ankylosing spondylitis genetic susceptibility loci overlap with those of other autoimmune diseases. Diseases are represented in columns, and ankylosing spondylitis susceptibility loci are represented in rows. Shared susceptibility loci are colored green if effect size is concordant and purple if effect size is discordant. Data are shown in Supplementary Table 8. SLE, systemic lupus erythematosus; T1D, type 1 diabetes.

tested on a reduced sample subset of individuals from the UK $(7,447$ cases and 11,479 controls). Considering loci with common variant associations achieving genome-wide significance, we identified six that harbored rare SNPs that were disease associated $(P<$ $5 \times 10^{-3}$; Supplementary Table 11). These associations remained significant after conditioning on the common variants, and variants at three loci (IL23R, TYK2 and KEAP1)

$H L A-A^{*} 0201\left(r^{2}<0.01\right)$, and, thus, this association is not a manifestation of LD with $H L A-B^{\star} 27$.

\section{Overlap with other immune-mediated diseases}

Considering the loci associated with ankylosing spondylitis in this study, we found substantial overlap with other immune-mediated diseases (Fig. 3 and Supplementary Table 8), notably, inflammatory bowel disease (either Crohn's disease or ulcerative colitis) and celiac disease. Ankylosing spondylitis-associated loci were associated with the same SNP in the same direction of association at 12 loci shared with Crohn's disease, at 11 loci shared with ulcerative colitis and at 6 of 7 loci shared with celiac disease. Overlap of associated loci with other diseases was not as marked, including for rheumatoid arthritis (one concordant, one discordant), psoriasis (two concordant), multiple sclerosis (three concordant, three discordant) and type 1 diabetes (two concordant, two discordant).

\section{Refinement of disease associations and secondary signals}

In the design of the Immunochip, eight loci already known to be associated with ankylosing spondylitis were selected for fine mapping. Compared with available ankylosing spondylitis GWAS data, the current study had greater marker density at these loci and larger sample size (from 3,023 cases and 8,779 controls to 10,619 cases and 15,145 controls). Nonetheless, for most loci studied, the disease-associated region was not substantially narrowed; less than $10 \%$ narrowing of the region was observed for four of the eight loci that were fine mapped (Supplementary Table 9). This suggests that, for many loci associated with common variants, the extent of $\mathrm{LD}$ at the locus will be too great to permit substantial refinement of the locus using sample sizes and marker densities of the magnitude employed here.

Two or more independent signals were identified at 12 of the 25 genome-wide significant loci $\left(P<5 \times 10^{-4}\right.$; Table 2 and Supplementary Fig. 9), including 1 locus (ERAP1 on chromosome 5 q15) with 3 associated haplotypes (Supplementary Fig. 3). This is a similar proportion to that found in celiac disease (13 of 36 loci) ${ }^{10}$. Taken together, these secondary signals contribute $0.75 \%$ of the heritability of ankylosing spondylitis (Supplementary Table 10).

\section{Rare variants}

Because of the likelihood of population stratification affecting rare variant associations, rare variant associations $(\mathrm{MAF}<1 \%)$ were only remained significant after controlling for the number of variants studied per locus. Four loci had exonic rare variant associations in the absence of a common variant association $\left(P<5 \times 10^{-5}\right)(K L K B 1$, RAD50, PRDM1 and DYRK4; Supplementary Table 11b). The rare variant with the largest effect was a predicted splice-site variant in TYK2 (rs280518: OR = 7.7, $P=0.002$ ).

\section{Comparisons across ancestry groups}

The power of our East Asian case-control cohort was much lower than for our European cohort; nonetheless, at least nominal association $(P<0.01)$ was detected in East Asians at 13 of the 23 loci for which we identified associations at genome-wide significance in the overall data set (Table 1).

At some loci, association was seen in both East Asians and Europeans but with different SNPs. At IL23R, the primary associated variant in Europeans, rs11209026, was not polymorphic in East Asians, as we and others have previously reported ${ }^{25}$. However, association was observed at $I L 23 R$ with a low-frequency nonsynonymous SNP in East Asians (rs76418789: p.Gly149Arg, OR $=1.5, P=8.2 \times$ $10^{-4}$ ). The minor, protective allele was predicted to be deleterious by both SIFT ${ }^{26}$ and PolyPhen analysis ${ }^{27}$. The same SNP was also nominally associated with ankylosing spondylitis in Europeans $(P=0.01)$. The MAF of rs76418789 was 10 times greater in East Asians than in Europeans (East Asians, 3.7\%; Europeans, 0.34\%). Of the other loci associated with ankylosing spondylitis in Europeans but not in East Asians, only at $\mathrm{BACH} 2$ was the key associated variant present at a much lower frequency in East Asians (rs17765610; MAF of $11.8 \%$ in Europeans and $1.8 \%$ in East Asians), suggesting that, at most loci with discordant association between ancestry groups, this was not due to differences in the population frequency of the associated SNP.

At PTGER4, association in Europeans peaked $155 \mathrm{~kb} \mathrm{5'}$ of the gene (peak associated SNP, rs12186979: $\mathrm{OR}=1.1, P=5.4 \times 10^{-6}$ ), whereas the peak of association in East Asians was in intron 2 of the gene $\left(\right.$ rs13354346: $\left.\mathrm{OR}=1.3, P=1.5 \times 10^{-5}\right)($ Supplementary Fig. 5).

\section{DISCUSSION}

This study confirmed the association of 12 of the 13 previously reported loci associated with ankylosing spondylitis in Europeans and identified 13 additional loci at genome-wide significance. We found no independent support for two loci previously reported to be associated with ankylosing spondylitis in Han Chinese, suggesting that the original 
associations of these loci may have been false positives. Additional associated haplotypes were identified at 13 loci, increasing the total number of distinct ankylosing spondylitis associations to 43 .

These findings highlight the role of some major biological pathways in the pathogenesis of ankylosing spondylitis, including the IL-23 pathway, gut immunity, T-lymphocyte differentiation or activation, and peptide processing before HLA class I presentation.

We identified three new ankylosing spondylitis-associated genes (TYK2, IL27 and IL6R) with known effects on the IL-23 pathway. TYK2 is a member of the Janus kinase family of intracellular signaling proteins and is involved in signal transduction from IL-23R, as well as other cytokine receptors, including those in interferon (IFN)- $\alpha$, IFN- $\beta$, IL-6, IL-10 and IL-12 signaling. Common TYK2 variants are also associated with Crohn's disease ${ }^{28}$ and psoriasis ${ }^{29}$. A different, rare TYK2 variant, rs34536443, is associated with multiple sclerosis ${ }^{30}$. IL-6 signaling through IL-6R has diverse proinflammatory effects. In combination with transforming growth factor (TGF)- $\beta$, it influences the ratio of $\mathrm{T}_{\mathrm{H}} 17$ to regulatory $\mathrm{T}\left(\mathrm{T}_{\text {reg }}\right)$ cells, promoting the differentiation of $\mathrm{T}_{\mathrm{H}} 17$ cells from naive $\mathrm{T}$ cells and inhibiting TGF- $\beta$-induced differentiation into $\mathrm{T}_{\text {reg }}$ cells ${ }^{31}$. Previous studies have reported no increase in $\mathrm{T}_{\mathrm{H}} 17$ lymphocyte counts in ankylosing spondylitis, suggesting that the IL6R association with ankylosing spondylitis operates through mechanisms other than effects on $\mathrm{T}_{\mathrm{H}} 17$ lymphocytes ${ }^{32,33}$. Whether IL- 6 has a role in the differentiation or activation of non-canonical cellular sources of IL-17 such as $\gamma \delta$ T cells, NK cells, neutrophils and mast cells, which have been implicated in ankylosing spondylitis, is unclear. IL-27 potentiates the differentiation of $\mathrm{CD} 4^{+} \mathrm{T}_{\mathrm{H}} 1$ cells, while suppressing the differentiation of $\mathrm{T}$ helper $2\left(\mathrm{~T}_{\mathrm{H}} 2\right)$ and $\mathrm{T}_{\mathrm{H}} 17$ cells. IL27 has previously been associated with both Crohn's disease and type 1 diabetes (Supplementary Table 8), with the association in type 1 diabetes being in the opposite direction to that observed in both ankylosing spondylitis and Crohn's disease.

At $I L 23 R$, we identified rare variant associations with ankylosing spondylitis in addition to the two known common variant haplotypes at this locus. Although one of these haplotypes has been shown to be due to association with the rs 11209026 coding SNP 6,25 , it is not clear from these genetic studies whether the second haplotype tagged by the intergenic SNP rs12141575 influences ankylosing spondylitis through effects on IL23R or IL12RB2. IL12RB2 encodes one of the two subunits of IL-12R, the stimulation of which drives CD4 $4^{+}$lymphocyte differentiation toward the $\mathrm{T}_{\mathrm{H}} 1$ lineage and away from the $\mathrm{T}_{\mathrm{H}} 17$ lymphocyte phenotype.

We identified six ankylosing spondylitis-associated genes that were also associated either with variation in $\mathrm{CD} 8^{+}$lymphocyte counts (EOMES, IL7R, RUNX3 and ZMIZ1) or CD4 ${ }^{+}$lymphocyte counts (BACH2 and SH2B3). EOMES encodes eomesodermin, a transcription factor involved in $\mathrm{CD}^{+} \mathrm{T}$ cell differentiation whose expression is induced by RUNX3 (refs. 34-36). Where eomesodermin is deficient, $\mathrm{CD}^{+} \mathrm{T}$ cells have been shown to express IL-17 (ref. 37). IL-7 acts through IL-7R to induce RUNX3 expression in developing T cells, in turn favoring differentiation toward the $\mathrm{CD}^{+} \mathrm{T}^{+}$cell lineage ${ }^{38}$. ZMIZ1 is a transcriptional coactivator of the protein inhibitor of activated STAT (PIAS)-like family and thus may have effects on STAT-mediated cytokine signaling. ZMIZ1 has recently been shown to cooperate with activating NOTCH1 mutations in inducing $\mathrm{T}$ cell acute lymphoblastic leukemia, consistent with it having a role in $\mathrm{T}$ cell differentiation ${ }^{39}$. Whether these genes affect risk of ankylosing spondylitis directly through effects on $\mathrm{CD}^{+} \mathrm{T}$ cell differentiation is unclear. For example, although the risk haplotype at RUNX3 is associated with lower CD8 ${ }^{+}$ T cell counts, at $I L 7 R$, the opposite phenotype is observed, suggesting that the mechanisms involved are more complex than a simple effect on
$\mathrm{CD}^{+} \mathrm{T}$ cell counts. IL-7 treatment has been shown to increase $\mathrm{T}_{\mathrm{H}} 17$ lymphocyte counts, and it may be that the association of $I L 7 R$ with ankylosing spondylitis operates directly through such an effect ${ }^{40}$.

One of the genes associated with both ankylosing spondylitis and $\mathrm{CD} 4{ }^{+}$lymphocyte counts, $\mathrm{BACH} 2$, encodes a B cell-specific transcription factor with diverse effects on $\mathrm{B}$ cell differentiation and function ${ }^{41}$; association of ankylosing spondylitis with $\mathrm{CD} 4^{+}$lymphocyte counts may thus be an indirect effect mediated by B cells. This is particularly noteworthy given the recent evidence suggesting that rituximab, a B cell-targeted therapy, may have beneficial effects in ankylosing spondylitis ${ }^{42}$. SH2B3 (also known as LNK) encodes an adaptor protein involved in $\mathrm{T}$ cell receptor signaling ${ }^{43}$. $\mathrm{CD} 8^{+}$lymphocytes are activated by the interaction of MHC class I peptides with their T cell receptors and may in turn become cytotoxic or memory $\mathrm{T}$ cells.

The association of four aminopeptidases involved in peptide trimming before HLA class I presentation is particularly noteworthy. We have shown here and previously that genetic variants associated with reduced function of ERAP1 and loss of expression of ERAP2 are protective for ankylosing spondylitis. Whether $L N P E P$ is also involved is uncertain, and identification of the key associated variants at NPEPPS will require further studies. It is possible that these genes operate in ankylosing spondylitis through a quantitative effect on HLA class I peptide presentation or a qualitative effect on the peptide repertoire presented. Downregulation of ERAP1 (refs. 44,45) and ERAP2 (ref. 19) expression has been shown to reduce cell surface expression of HLA class I molecules. ERAP1 preferentially cleaves hydrophobic amino acids, whereas ERAP2 preferentially cleaves basic residues. ERAP1-ERAP2 heterodimers may thus act in concert, particularly in cleaving longer peptides ${ }^{45}$. It has been suggested that misfolding of nascent HLA-B ${ }^{\star} 27$ in the ER, leading to ER stress, may be involved in the pathogenesis of ankylosing spondylitis ${ }^{46}$. It is also possible that, by influencing the quantity of peptide available during HLA-B ${ }^{\star} 27$ folding, ERAP1 and ERAP2 variants associated with disease risk slow the rate of this folding, thereby increasing ER stress.

In this study, over one-third of loci with common variant associations were found to harbor more than one disease-associated haplotype. Identifying these additional haplotypes increased the proportion of genetic variance explained in ankylosing spondylitis and, more notably, led to valuable biological insights. For example, association of ankylosing spondylitis with SNPs on chromosome 12p13 has previously been reported, although it was not clear whether the association was primarily with TNFRSF1A or LTBR, both plausible candidate genes $^{6,7,47}$. The current study shows that there are two signals at this locus, one in TNFRSF1A and the other in LTBR. The primary associated SNP at TNFRSF1A (rs1860545) is in strong LD with a multiple sclerosis-associated SNP, $\operatorname{rs} 1800696\left(r^{2}=0.96, D^{\prime}=0.98\right)$ but with the opposite direction of association. rs 1800696 has recently been shown to lead to the splicing out of exon 6 of TNFR1, resulting in loss of the transmembrane domain ${ }^{48}$. The resulting protein acts as a soluble decoy receptor for TNF, akin to the TNF inhibitor drug etanercept. TNF inhibitors are highly effective therapeutic drugs in ankylosing spondylitis, but their use can lead to induction or exacerbation of multiple sclerosis. The association with ankylosing spondylitis suggests the possibility that disease activity and response to TNF inhibitor therapy may be affected by this SNP.

Comparison of the genetic associations of ankylosing spondylitis with other diseases reinforces the considerable overlap with Crohn's disease, ulcerative colitis and ankylosing spondylitis. Ankylosing spondylitis frequently complicates inflammatory bowel disease (both Crohn's disease and ulcerative colitis), and increased cofamiliality with inflammatory bowel disease has been demonstrated ${ }^{49,50}$ 
suggesting shared etiopathogenesis. Overlapping genetic susceptibility between ankylosing spondylitis and inflammatory bowel disease has previously been reported ${ }^{51,52}$. The genes involved include many with effects on the IL-23 pathway, supporting the notion of this being a key pathway in the pathogenesis of these conditions, most likely through effects on gut mucosal immunology. However, the major loci for each disease are not shared, with ankylosing spondylitis showing no association with NOD2 or ATG16L1 and neither Crohn's disease nor ulcerative colitis showing association with $H L A-B^{\star} 27$. This suggests that these disease-specific loci contribute to the organ and tissue specificity of the diseases with which they are associated, whereas the IL-23 pathway is involved in the core immunological pathway underlying all these conditions.

We also identified associations with three loci encoding $G$ proteincoupled receptors, including GPR35, GPR37 and GPR65, and a fourth (GPR25) is close to $K I F 21 B$, an established ankylosing spondylitis locus where the key associated variants are not yet defined. The functions of these genes and their ligands are not well established. GPR35 is reported to act as a receptor for 2-acyl lysophosphatidic acid, and GPR65 is reported to be a receptor for glycosphingolipids and protons; the ligands for GPR25 and GPR37 are not known. GPR65 has previously been associated with Crohn's disease ${ }^{53}$ and multiple sclerosis $^{54}$. The mouse homolog of GPR65, T cell death-associated gene 8, inhibits proinflammatory cytokine production (including of TNF- $\alpha$ and IL-6) in acidic conditions ${ }^{55}$, suggesting a potential mechanism in diverse autoimmune diseases. However, it also has anti-apoptotic effects and an ability to activate not only cyclic AMP (cAMP) intracellular signaling but also other pathways, including mitogen-activated protein kinase (MAPK) and MEK/ERK signaling and thus is likely to have multiple functions ${ }^{56}$. Further research is needed into the functions of these genes and their roles in autoimmune diseases.

It has long been suspected that associations in the MHC region with ankylosing spondylitis are not completely explained by $H L A-B^{\star} 27$. The association of $H L A-A^{*} 0201$ with ankylosing spondylitis at genome-wide significance in both $H L A-B^{\star} 27$-positive and $H L A-B^{\star} 27$-negative cases confirms that suspicion. $H L A-A^{\star} 02$ has previously been reported to be associated with anterior uveitis complicating ankylosing spondylitis ${ }^{57}$ and is a risk factor for vitiligo ${ }^{58}$. $H L A-A^{*} 0201$ has a protective effect in multiple sclerosis ${ }^{54}$ and is a risk allele for type 1 diabetes ${ }^{59}$, but no $H L A-A$ association has previously been reported with ankylosing spondylitis itself.

In conclusion, we have increased the number of ankylosing spondylitis-associated loci to 31, identifying 13 new loci and 12 additional ankylosing spondylitis-associated haplotypes at 11 loci, bringing the total number of genetic signals independently associated with ankylosing spondylitis to 43 . These loci reinforce the mounting evidence that aberrant peptide processing before $\mathrm{MHC}$ class I presentation and alterations of the IL-23 pathway are key elements in the pathogenesis of ankylosing spondylitis.

URL. Haploxt, http://genome.sph.umich.edu/wiki/Haploxt.

\section{METHODS}

Methods and any associated references are available in the online version of the paper.

Note: Supplementary information is available in the online version of the paper.

\section{ACKNOWLEDGMENTS}

We thank all participating subjects with ankylosing spondylitis and healthy individuals who provided the DNA and clinical information necessary for this study. The Wellcome Trust Case Control Consortium 2 project is funded by the
Wellcome Trust (083948/Z/07/Z). We acknowledge use of the British 1958 Birth Cohort DNA collection, funded by the Medical Research Council (G0000934) and the Wellcome Trust (068545/Z/02), and of the UK National Blood Service controls, funded by the Wellcome Trust. We thank J.C. Barrett for contributing the design of the Immunochip and for helpful analytical discussion, as well as E. Gray, S. Bumpstead, D. Simpkin and the staff of the Wellcome Trust Sanger Institute Sample Management and Genotyping teams for their genotyping and analytical contributions. The Australo-Anglo-American Spondyloarthritis Consortium (TASC) study was funded by National Institute of Arthritis and Musculoskeletal and Skin Diseases (NIAMS) grants P01-052915 and R01-AR046208. Funding was also received from University of Texas at Houston Clinical and Translational Science Award (CTSA) UL1RR024188, Cedars-Sinai General Clinical Research Center (GCRC) grant MO1-RR00425, the Intramural Research Program, NIAMS, US National Institutes of Health and the Rebecca Cooper Foundation (Australia). This study was funded, in part, by Arthritis Research UK (grants 19536 and 18797), by the Wellcome Trust (grant 076113) and by the Oxford Comprehensive Biomedical Research Centre ankylosing spondylitis chronic disease cohort (theme A91202). We thank A. Harrison (University of Otago) for his contribution to the New Zealand ankylosing spondylitis cohort. H.X. was funded by National Natural Science Foundation of China grants 81020108029 and 30872339. Portuguese sample collection was performed by COnhecer a Realidade PORtuguesa sobre a Espondilite Anquilosante (CORPOREA Study Group), coordinated by F.M.P.-S. and supported by Bolsa Investigação da Sociedade Portuguesa de Reumatologia/ Schering-Plough 2007. The Spanish ankylosing spondylitis case collection was supported by Spanish grant FICYT PC-10-70-Fondos FEDER European Union. The Spondyloarthritis Research Consortium of Canada (SPARCC) was funded by a National Research Initiative Award from the Arthritis Society (Canada). French sample collection was performed by the Groupe Française d'Etude Génétique des Spondylarthrites, coordinated by R. Said-Nahal and funded by Agence Nationale de Recherche GEnetics, Microbiota, Inflammation and Spondyloarthritis (GEMISA) grant ANR-10-MIDI-0002. We thank the Norwegian Bone Marrow Donor registry for providing data from healthy Norwegian controls. W.P.M. is a Medical Scientist of Alberta Innovates-Health Solutions. The Psoriatic Arthritis Program is supported by the Krembil Foundation and the Arthritis Society. P.C.R. is funded by the National Health and Medical Research Council (Australia) (NHMRC) and Arthritis Australia. J.Y. is supported by NHMRC grants 613672 and 1011506. M. Ward is supported by the Intramural Research Program, NIAMS, US National Institutes of Health. D.E. is supported by the research council of Ghent University and by the Fund for Scientific Research Flanders. M.A.B. is funded by a National Health and Medical Research Council (Australia) Senior Principal Research Fellowship, and support for this study was received from a National Health and Medical Research Council (Australia) program grant (566938) and project grant (569829) and from the Australian Cancer Research Foundation and the Rebecca Cooper Medical Research Foundation. We thank A. Gardiner and the Brisbane Convention and Exhibition Centre for their assistance in preparing the manuscript. We are also very grateful for the invaluable support received from the National Ankylosing Spondylitis Society (UK) and the Spondyloarthritis Association of America in case recruitment. Additional financial and technical support for subject recruitment was provided by the NIHR Oxford Musculoskeletal Biomedical Research Unit and NIHR Thames Valley Comprehensive Local Research and by an unrestricted educational grant from Abbott Laboratories.

\section{AUTHOR CONTRIBUTIONS}

J. Hadler, K.C., K.P. and J. Harris performed genotyping. A.C., P.C.R., T.K., P.L., J.Y., M.A.B. and D.M.E. performed statistical analyses. J.P.P., S.L., K.B.J., S.-C.S., M. Weisman, M. Ward, X.Z., H.-J.G., G.C., J.N., B.A.L., Ø.F., J.T., K.L., L.J., Y.L., X.W., L.A.B., D.E., R.B.-V., S.S., L.A., C.F., J.L., N.H., J. Mulero, J.L.F.-S., M.A.G.-G., C.L.-L., P. Deloukas, P. Donnelly, P.B., K.G., H.G., D.D.G., P.R., W.P.M., H.X., J.B.A.C., I.E.v.d.H.-B., C.-T.C., R.V.-O., C.R.-S., I.M.H., F.M.P.-S., R.D.I., V.V., J. Martin, M.B., J.D.R. and T.-H.K. all contributed to subject recruitment and study design. A.C., M.A.B., D.M.E. and B.P.W. wrote the manuscript, and all authors contributed to manuscript drafting and reviewed the final manuscript. T.J.K. performed cell count and IL-6R studies in ankylosing spondylitis cases and controls. M.A.F. performed GWAS of cell counts in controls.

\section{COMPETING FINANCIAL INTERESTS}

The authors declare no competing financial interests.

Reprints and permissions information is available online at http://www.nature.com/ reprints/index.html.

1. Braun, J., Listing, J. \& Sieper, J. Overestimation of the prevalence of ankylosing spondylitis in the Berlin study: comment on the article by Braun et al-Reply. Arthritis Rheum. 52, 4049-4050 (2005). 
2. Ng, S.C. et al. Epidemiology of spondyloarthritis in the People's Republic of China: review of the literature and commentary. Semin. Arthritis Rheum. 37, 39-47 (2007).

3. Brown, M.A., Laval, S.H., Brophy, S. \& Calin, A. Recurrence risk modelling of the genetic susceptibility to ankylosing spondylitis. Ann. Rheum. Dis. 59, 883-886 (2000).

4. Brown, M.A. et al. Susceptibility to ankylosing spondylitis in twins: the role of genes, HLA, and the environment. Arthritis Rheum. 40, 1823-1828 (1997).

5. Burton, P.R. et al. Association scan of 14,500 nonsynonymous SNPs in four diseases identifies autoimmunity variants. Nat. Genet. 39, 1329-1337 (2007).

6. Evans, D.M. et al. Interaction between ERAP1 and HLA-B27 in ankylosing spondylitis implicates peptide handling in the mechanism for HLA-B27 in disease susceptibility. Nat. Genet. 43, 761-767 (2011).

7. Reveille, J.D. et al. Genome-wide association study of ankylosing spondylitis identifies non-MHC susceptibility loci. Nat. Genet. 42, 123-127 (2010).

8. Lin, Z. et al. A genome-wide association study in Han Chinese identifies new susceptibility loci for ankylosing spondylitis. Nat. Genet. 44, 73-77 (2012).

9. Cortes, A. \& Brown, M.A. Promise and pitfalls of the Immunochip. Arthritis Res. Ther. 13, 101 (2011).

10. Trynka, G. et al. Dense genotyping identifies and localizes multiple common and rare variant association signals in celiac disease. Nat. Genet. 43, 1193-1201 (2011).

11. Ferreira, M.A. et al. Identification of IL6R and chromosome $11 \mathrm{q} 13.5$ as risk loci for asthma. Lancet 378, 1006-1014 (2011).

12. Melzer, D. et al. A genome-wide association study identifies protein quantitative trait loci (pQTLs). PLoS Genet. 4, e1000072 (2008).

13. Mizuki, N. et al. Genome-wide association studies identify IL23R-IL12RB2 and IL10 as Behcet's disease susceptibility loci. Nat. Genet. 42, 703-706 (2010).

14. Remmers, E.F. et al. Genome-wide association study identifies variants in the MHC class I, IL10, and IL23R-IL12RB2 regions associated with Behcet's disease. Nat. Genet. 42, 698-702 (2010).

15. Ait Badi, M.A. et al. Skeletal manifestations in Behcet's disease. A report of 79 cases. Rev. Med. Interne 29, 277-282 (2008).

16. Harvey, D. et al. A common functional variant of endoplasmic reticulum aminopeptidase 2 (ERAP2) that reduces major histocompatibility complex class I expression is not associated with ankylosing spondylitis. Rheumatology 50, 1720-1721 (2011).

17. Tsui, F.W. et al. Association of an ERAP1-ERAP2 haplotype with familial ankylosing spondylitis. Ann. Rheum. Dis. 69, 733-736 (2010).

18. Evnouchidou, I. et al. A common single nucleotide polymorphism in Endoplasmic Reticulum Aminopeptidase 2 induces a specificity switch that leads to altered antigen processing. J. Immunol. 189, 2383-2392 (2012).

19. Andrés, A.M. et al. Balancing selection maintains a form of ERAP2 that undergoes nonsense-mediated decay and affects antigen presentation. PLoS Genet. 6, e1001157 (2010)

20. Lévy, F. et al. The final N-terminal trimming of a subaminoterminal prolinecontaining HLA class I-restricted antigenic peptide in the cytosol is mediated by two peptidases. J. Immunol. 169, 4161-4171 (2002).

21. Xia, Z. et al. A 17q12 allele is associated with altered NK cell subsets and function. J. Immunol. 188, 3315-3322 (2012).

22. Trynka, G. et al. Chromatin marks identify critical cell types for fine mapping complex trait variants. Nat. Genet. 45, 124-130 (2013).

23. ENCODE Project Consortium. An integrated encyclopedia of DNA elements in the human genome. Nature 489, 57-74 (2012).

24. Ferreira, M.A. et al. Quantitative trait loci for CD4:CD8 lymphocyte ratio are associated with risk of type 1 diabetes and HIV-1 immune control. Am. J. Hum. Genet. 86, 88-92 (2010).

25. Davidson, S.I. et al. Association of ERAP1, but not IL23R, with ankylosing spondylitis in a Han Chinese population. Arthritis Rheum. 60, 3263-3268 (2009).

26. Kumar, P., Henikoff, S. \& Ng, P.C. Predicting the effects of coding non-synonymous variants on protein function using the SIFT algorithm. Nat. Protoc. 4, 1073-1081 (2009).

27. Adzhubei, I.A. et al. A method and server for predicting damaging missense mutations. Nat. Methods 7, 248-249 (2010).

28. Sato, K et al. Strong evidence of a combination polymorphism of the tyrosine kinase 2 gene and the signal transducer and activator of transcription 3 gene as a DNA-based biomarker for susceptibility to Crohn's disease in the Japanese population. J. Clin. Immunol. 29, 815-825 (2009).

29. Strange, A. et al. A genome-wide association study identifies new psoriasis susceptibility loci and an interaction between HLA-C and ERAP1. Nat. Genet. 42, 985-990 (2010).

30. Ban, M. et al. Replication analysis identifies TYK2 as a multiple sclerosis susceptibility factor. Eur. J. Hum. Genet. 17, 1309-1313 (2009).

31. Bettelli, E. et al. Reciprocal developmental pathways for the generation of pathogenic effector $T_{H} 17$ and regulatory T cells. Nature 441, 235-238 (2006).

32. Kenna, T.J. et al. Enrichment of circulating interleukin-17-secreting interleukin-23 receptor-positive $\gamma / \delta$ T cells in patients with active ankylosing spondylitis. Arthritis Rheum. 64, 1420-1429 (2012).
33. Appel, $\mathrm{H}$, et al. Analysis of $1 \mathrm{~L}-17^{+}$cells in facet joints of patients with spondyloarthritis suggests that the innate immune pathway might be of greater relevance than the Th17-mediated adaptive immune response. Arthritis Res. Ther. 13, R95 (2011)

34. Pearce, E.L. et al. Control of effector $\mathrm{CD}^{+} \mathrm{T}$ cell function by the transcription factor Eomesodermin. Science 302, 1041-1043 (2003).

35. Yagi, R. et al. The transcription factor GATA3 actively represses RUNX3 proteinregulated production of interferon- $\gamma$. Immunity 32, 507-517 (2010).

36. Cruz-Guilloty, F. et al. Runx3 and T-box proteins cooperate to establish the transcriptional program of effector CTLs. J. Exp. Med. 206, 51-59 (2009).

37. Intlekofer, A.M. et al. Anomalous type 17 response to viral infection by CD8 T cells lacking T-bet and eomesodermin. Science 321, 408-411 (2008).

38. Park, J.H. et al. Signaling by intrathymic cytokines, not $T$ cell antigen receptors, specifies CD8 lineage choice and promotes the differentiation of cytotoxic-lineage T cells. Nat. Immunol. 11, 257-264 (2010).

39. Rakowski, L.A. et al. Convergence of the ZMIZ1 and NOTCH1 pathways at C-MYC in acute T lymphoblastic leukemias. Cancer Res. 73, 930-941 (2013).

40. Hartgring, S.A., Willis, C.R., Bijlsma, J.W., Lafeber, F.P. \& van Roon, J.A. Interleukin 7 aggravated joint inflammation and tissue destruction in collagen-induced arthritis is associated with T-cell and B-cell activation. Arthritis Res. Ther. 14, R137 (2012).

41. Muto, A. et al. Bach2 represses plasma cell gene regulatory network in B cells to promote antibody class switch. EMBO J. 29, 4048-4061 (2010).

42. Song, I.H. et al. Different response to rituximab in tumor necrosis factor blockernaive patients with active ankylosing spondylitis and in patients in whom tumor necrosis factor blockers have failed: a twenty-four-week clinical trial. Arthritis Rheum. 62, 1290-1297 (2010).

43. Huang, X., Li, Y., Tanaka, K., Moore, K.G. \& Hayashi, J.I. Cloning and characterization of Lnk, a signal transduction protein that links T-cell receptor activation signal to phospholipase $\mathrm{C} \gamma 1$, Grb2, and phosphatidylinositol 3-kinase. Proc. Natl. Acad. Sci. USA 92, 11618-11622 (1995).

44. Evnouchidou, I. et al. Cutting Edge: Coding single nucleotide polymorphisms of endoplasmic reticulum aminopeptidase 1 can affect antigenic peptide generation in vitro by influencing basic enzymatic properties of the enzyme. J. Immunol. 186, 1909-1913 (2011).

45. Saveanu, L. et al. Concerted peptide trimming by human ERAP1 and ERAP2 aminopeptidase complexes in the endoplasmic reticulum. Nat. Immunol. 6 , 689-697 (2005).

46. Colbert, R.A. et al. HLA-B27 misfolding activates the IL-23/IL-17 axis via the unfolded protein response in transgenic rats: evidence for a novel mechanism of inflammation. Arthritis Rheum. 1283, S515 (2007).

47. Karaderi, T. et al. Evidence of genetic association between TNFRSF1A encoding the p55 tumour necrosis factor receptor, and ankylosing spondylitis in UK Caucasians. Clin. Exp. Rheumatol. 30, 110-113 (2012).

48. Gregory, A.P. et al. TNF receptor 1 genetic risk mirrors outcome of anti-TNF therapy in multiple sclerosis. Nature 488, 508-511 (2012)

49. Hemminki, K., Li, X., Sundquist, K. \& Sundquist, J. Familial association of inflammatory bowel diseases with other autoimmune and related diseases. Am. J. Gastroenterol. 105, 139-147 (2010).

50. Thjodleifsson, B., Geirsson, A.J., Bjornsson, S. \& Bjarnason, I. A common genetic background for inflammatory bowel disease and ankylosing spondylitis: a genealogic study in Iceland. Arthritis Rheum. 56, 2633-2639 (2007).

51. Danoy, P. et al. Association of variants at $1 \mathrm{q} 32$ and STAT3 with ankylosing spondylitis suggests genetic overlap with Crohn's disease. PLoS Genet. 6, e1001195 (2010).

52. Laukens, D. et al. Evidence for significant overlap between common risk variants for Crohn's disease and ankylosing spondylitis. PLOS ONE 5, e13795 (2010).

53. Franke, A. et al. Genome-wide meta-analysis increases to 71 the number of confirmed Crohn's disease susceptibility loci. Nat. Genet. 42, 1118-1125 (2010).

54. Sawcer, S. et al. Genetic risk and a primary role for cell-mediated immune mechanisms in multiple sclerosis. Nature 476, 214-219 (2011).

55. Onozawa, Y. et al. Activation of T cell death-associated gene 8 regulates the cytokine production of T cells and macrophages in vitro. Eur. J. Pharmacol. 683, 325-331 (2012).

56. Ryder, C., McColl, K., Zhong, F. \& Distelhorst, C.W. Acidosis promotes Bcl-2 family mediated evasion of apoptosis: involvement of acid-sensing $\mathrm{G}$ protein-coupled receptor GPR65 signaling to MEK/ERK. J. Biol. Chem. 287, 27863-27875 (2012).

57. Khan, M.A., Kushner, I. \& Braun, W.E. Association of HLA-A2 with uveitis in HLA-B27 positive patients with ankylosing spondylitis. J. Rheumatol. 8, 295-298 (1981).

58. Liu, J.B. et al. Association of vitiligo with HLA-A2: a meta-analysis. J. Eur. Acad. Dermatol. Venereol. 21, 205-213 (2007).

59. Noble, J.A. et al. HLA class I and genetic susceptibility to type 1 diabetes: results from the Type 1 Diabetes Genetics Consortium. Diabetes 59, 2972-2979 (2010) 
Adrian Cortes ${ }^{1}$, Johanna Hadler ${ }^{1}$, Jenny P Pointon ${ }^{2}$, Philip C Robinson ${ }^{1}$, Tugce Karaderi ${ }^{2}$, Paul Leo ${ }^{1}$, Katie Cremin ${ }^{1}$, Karena Pryce ${ }^{1}$, Jessica Harris ${ }^{1}$, Seunghun Lee ${ }^{3}$, Kyung Bin Joo ${ }^{3}$, Seung-Cheol Shim ${ }^{4}$, Michael Weisman ${ }^{5}$, Michael Ward ${ }^{6}$, Xiaodong Zhou ${ }^{7}$, Henri-Jean Garchon ${ }^{8,9}$, Gilles Chiocchia ${ }^{8}$, Johannes Nossent ${ }^{10,11}$, Benedicte A Lie ${ }^{12,13}$, Øystein Førre ${ }^{14}$, Jaakko Tuomilehto ${ }^{15-17}$, Kari Laiho ${ }^{18}$, Lei Jiang ${ }^{19}$, Yu Liu ${ }^{19}$, Xin Wu ${ }^{19}$, Linda A Bradbury ${ }^{1}$, Dirk Elewaut ${ }^{20}$, Ruben Burgos-Vargas ${ }^{21}$, Simon Stebbings ${ }^{22}$, Louise Appleton ${ }^{2}$, Claire Farrah ${ }^{2}$, Jonathan Lau ${ }^{2}$, Tony J Kenna ${ }^{1}$, Nigil Haroon ${ }^{23}$, Manuel A Ferreira ${ }^{24}$, Jian Yang ${ }^{1}$, Juan Mulero ${ }^{25}$, Jose Luis Fernandez-Sueiro ${ }^{26}$, Miguel A Gonzalez-Gay ${ }^{27}$, Carlos Lopez-Larrea ${ }^{28,29}$, Panos Deloukas ${ }^{30}$, Peter Donnelly ${ }^{31}$, Australo-Anglo-American Spondyloarthritis Consortium (TASC) ${ }^{32}$, Groupe Française d'Etude Génétique des Spondylarthrites (GFEGS) ${ }^{32}$, Nord-Trøndelag Health Study (HUNT) ${ }^{32}$, Spondyloarthritis Research Consortium of Canada (SPARCC) ${ }^{32}$, Wellcome Trust Case Control Consortium 2 (WTCCC2) $^{32}$, Paul Bowness ${ }^{2}$, Karl Gafney ${ }^{33}$, Hill Gaston ${ }^{34}$, Dafna D Gladman ${ }^{35-37}$, Proton Rahman ${ }^{38}$, Walter P Maksymowych ${ }^{39}$, Huji Xu ${ }^{19}$, J Bart A Crusius ${ }^{40}$, Irene E van der Horst-Bruinsma ${ }^{41}$, Chung-Tei Chou ${ }^{42,43}$, Raphael Valle-Oñate ${ }^{44}$, Consuelo Romero-Sánchez ${ }^{44}$, Inger Myrnes Hansen ${ }^{45}$, Fernando M Pimentel-Santos ${ }^{46}$, Robert D Inman ${ }^{23}$, Vibeke Videm ${ }^{47,48}$, Javier Martin ${ }^{49}$, Maxime Breban ${ }^{8,9}$, John D Reveille ${ }^{7}$, David M Evans ${ }^{50}$, Tae-Hwan Kim ${ }^{3}$, Bryan Paul Wordsworth ${ }^{2}$ \& Matthew A Brown ${ }^{1}$

${ }^{1}$ University of Queensland Diamantina Institute, Translational Research Institute, Brisbane, Queensland, Australia. ${ }^{2}$ National Institute for Health Research (NIHR) Oxford Musculoskeletal Biomedical Research Unit, Nuffield Orthopaedic Centre, Headington, Oxford, UK. ${ }^{3}$ Department of Rheumatology, Hanyang University Hospital for Rheumatic Diseases, Seoul, Republic of Korea. ${ }^{4}$ Department of Medicine, Division of Rheumatology, Eulji University Hospital, Daejeon, Republic of Korea. ${ }^{5}$ Department of Medicine/Rheumatology, Cedars-Sinai Medical Center, Los Angeles, California, USA. ${ }^{6}$ National Institute of Arthritis and Musculoskeletal and Skin Diseases, US National Institutes of Health, Bethesda, Maryland, USA. ${ }^{7}$ Rheumatology and Clinical Immunogenetics, University of Texas Health Science Center at Houston, Houston, Texas, USA. ${ }^{8}$ Institut Cochin, Université Paris-Descartes, Centre National de Recherche Scientifique (CNRS) Unité Mixte de Recherhce (UMR) 8104, Institut National de la Santé et de la Recherche Médicale (INSERM) U1016, Paris, France. ${ }^{9}$ Division of Rheumatology, Ambroise Paré Hospital, Assistance Publique-Hôpitaux de Paris, Versailles-Saint-Quentin en Yvelines University, Boulogne-Billancourt, France. ${ }^{10}$ University Hospital North Norway, Tromso, Norway. ${ }^{11}$ Division of Medicine, Royal Darwin Hospital, Darwin, Northern Territory, Australia. ${ }^{12}$ Department of Medical Genetics, University of Oslo and Oslo University Hospital, Oslo, Norway. ${ }^{13}$ Department of Immunology, Oslo University Hospital, Oslo, Norway. ${ }^{14}$ Department of Rheumatology, University Hospital Oslo, Oslo, Norway. ${ }^{15}$ Centre for Vascular Prevention, Danube-University Krems, Krems, Austria. ${ }^{16}$ Diabetes Prevention Unit, National Institute for Health and Welfare, Helsinki, Finland. ${ }^{17}$ King Abdulaziz University, Jeddah, Saudi Arabia. ${ }^{18}$ Paijat-Hame Central Hospital, Lahti, Finland. ${ }^{19}$ Department of Rheumatology and Immunology, Shanghai Changzheng Hospital, Second Military Medical University, Shanghai, China. ${ }^{20}$ Department of Rheumatology, Ghent University Hospital, Ghent, Belgium. ${ }^{21}$ Department of Rheumatology, Hospital General de México, Faculty of Medicine, Universidad Nacional Autónoma de México, Mexico City, México. ${ }^{22}$ Department of Medicine, Dunedin School of Medicine, University of Otago, Dunedin, New Zealand. ${ }^{23}$ Division of Rheumatology, Toronto Western Hospital, Toronto, Ontario, Canada. ${ }^{24}$ Queensland Institute of Medical Research, Royal Brisbane Hospital, Herston, Queensland, Australia. ${ }^{25}$ Rheumatology Department, Hospital Puerta de Hierro, Madrid, Spain. ${ }^{26}$ Rheumatology Department, Complejo Hospitalario La Coruña, Instituto de Investigación Biomédica A Coruña (INIBIC), La Coruña, Spain. ${ }^{27}$ Rheumatology Department, Hospital Marqués de Valcecilla, Instituto de Formación e Investigación Marqués de Valcecillas (IFIMAV), Santander, Spain. 28Department of Immunology, Asturias Central University Hospital, Oviedo, Spain. ${ }^{29}$ Fundación Renal Iñigo Alvarez de Toledo, Madrid, Spain. 30 Wellcome Trust Sanger Institute, Cambridge, UK. ${ }^{31}$ Wellcome Trust Centre for Human Genetics, University of Oxford, Oxford, UK. ${ }^{2}$ Details appear in the Supplementary Note. ${ }^{33}$ Department of Rheumatology, Norfolk and Norwich University Hospital, Norwich, UK. ${ }^{34}$ Department of Medicine, University of Cambridge, Addenbrooke's Hospital, Cambridge, UK. ${ }^{35}$ Division of Rheumatology, University of Toronto, Toronto, Ontario, Canada. ${ }^{36}$ Toronto Western Research Institute, Toronto, Ontario, Canada. ${ }^{37}$ Psoriatic Arthritis Program, University Health Network, Toronto, Ontario, Canada. ${ }^{8}$ Memorial University of Newfoundland, St. John's, Newfoundland, Canada. ${ }^{39}$ Department of Medicine, University of Alberta, Edmonton, Alberta, Canada. ${ }^{40}$ Laboratory of Immunogenetics, Department of Medical Microbiology and Infection Control, VU University Medical Center, Amsterdam, The Netherlands. ${ }^{41}$ Department of Rheumatology, VU University Medical Center, Amsterdam, The Netherlands. ${ }^{42}$ Department of Medicine, Division of Allergy, Immunology, Rheumatology, Taipei Veterans General Hospital, Taipei, Taiwan. 43 School of Medicine, National YangMing University, Taipei, Taiwan. ${ }^{44}$ Spondyloarthropathy Group, Division of Rheumatology, Hospital Militar Central, Universidad de La Sabana, Bogotá, Colombia. ${ }^{45}$ Helgelandssykehuset, Mo i Rana, Norway. ${ }^{46}$ Chronic Diseases Research Centre (CEDOC), Faculdade de Ciências Médicas, Universidade Nova de Lisboa, Lisbon, Portugal. ${ }^{47}$ Department of Laboratory Medicine, Children's and Women's Health, Norwegian University of Science and Technology, Trondheim, Norway. ${ }^{48}$ Department of Immunology and Transfusion Medicine, Trondheim University Hospital, Trondheim, Norway. ${ }^{49}$ Instituto de Parasitología y Biomedicina López-Neyra, Consejo Superior de Investigaciones Científicas, Granada, Spain. ${ }^{50}$ Medical Research Council (MRC) Centre for Causal Analyses in Translational Epidemiology, School of Social and Community Medicine, Bristol, UK. Correspondence should be addressed to M.A.B. (matt.brown@uq.edu.au). 


\section{ONLINE METHODS}

Samples. All cases had definite ankylosing spondylitis according to the modified New York criteria ${ }^{60}$. Written informed consent was obtained from all cases with approval from the relevant research ethics authorities at each participating center. A total of 12,252 DNA samples passed genotyping control filters. The case collection consisted of 10,417 individuals of European ancestry, 1,560 of Asian ancestry and 275 from Latin America (Colombia and Mexico). Of these, 2,425 cases of European ancestry have previously been reported in GWAS.

We obtained 12,338 controls of European ancestry, 1,570 of East Asian ancestry and 445 from Latin America. These included shared controls from the UK 1958 Birth Cohort, the UK Blood Services Common Controls and the United States and from participating centers from France, The Netherlands, Norway, Spain, Mexico, Colombia, China, Taiwan and Korea.

Genotyping. Samples were genotyped using the Immunochip, an Illumina Infinium platform, according to the manufacturer's recommendations. Control samples from the UK were genotyped at the Sanger Centre (Hinxton, Cambridge, UK) and at the University of Virginia (Charlottesville, Virginia, USA), control samples from the United States were genotyped at the Feinstein Institute (New York, New York, USA), and all other controls and all cases were genotyped at the University of Queensland Diamantina Institute (Brisbane, Queensland, Australia). Bead intensity data were processed and normalized for each sample in GenomeStudio; data for successfully genotyped samples were extracted, and genotypes were called within collections using optiCall ${ }^{61}$. NCBI Build 36 (hg18) mapping was used (Illumina manifest file Immuno_ BeadChip_11419691_B.bpm).

SNPs rs4552569, rs13210693 and rs17095830 were genotyped in 2,998 East Asian cases and 5,547 East Asian controls using TaqMan probes according to the manufacturer's instructions.

The genotyping accuracy of seven disease-associated SNPs with MAF $<1 \%$ was confirmed by custom TaqMan genotyping assays. Taking into consideration the five successfully developed assays, genotypes were completely concordant with Immunochip genotyping. Assays could not be developed for two SNPs (rs280518 in TYK2 and rs75430612 in IL2R2). However, given the clean clustering achieved for these SNPs in microarray genotyping and the fact that they had previously been reported, it is likely that they represent true positives.

Data quality control. We first excluded, for each of the collections separately, SNPs with call rate below $95 \%$ or with a Hardy-Weinberg equilibrium $P$ value of $<1 \times 10^{-6}$ in controls, as well as samples with call rate below $95 \%$. For the overlapping SNPs, we performed pairwise missingness tests between the collections and removed all SNPs with differential missingness $\left(P<1 \times 10^{-7}\right)$. After merging data sets, SNPs with call rate below $98 \%$ and samples with call rate below $98 \%$ were removed. A total of 128,935 SNPs were analyzed. Cluster plots were visually inspected for all SNPs used to inform conclusions.

The origins of samples of European and Asian ancestry were confirmed by principal-component analysis. Immunochip data were merged with genotype information from seven HapMap 3 populations (CEU, TSI, YRI, MEX, JPT, $\mathrm{CHD}$ and $\mathrm{CHB}$ ), and samples were identified as European or East Asian on the basis of their projection onto the first five principal components of genetic variation (Supplementary Figs. 10-12). Ancestry outliers were removed by assigning samples to an ancestry group using a model-based unsupervised clustering approach ${ }^{62}$. A second round of principal-component analysis was performed on the European and Asian populations independently to better resolve ancestry differences within the cohorts. In both cases, only the first principal component was correlated with the places of origin of the samples, and only in the European collection was the principal component marginally correlated with case-control status $(P=0.016)$. The projection of the samples analyzed onto the first two principal components is shown in Supplementary Figures 3 and 4.

Duplicated samples (intentional or unintentional) and those showing cryptic relatedness were assessed for the European and Asian cohorts separately by calculating identity by descent using PLINK (v1.07) ${ }^{63}$. For each pair of related samples (PI_HAT $>0.2$ ), the sample with the lower call rate was removed, and, where the pair involved a case and a control with similar call rates (both above 98\%), cases were preferentially selected for inclusion.
Association analysis. Association analysis and population stratification control was performed using linear mixed models as implemented in FaST-LMM (v1.05) ${ }^{64}$. For each chromosome, a relationship matrix was constructed with all SNPs, excluding those in the chromosome being analyzed and in the MHC region. Conditional analysis for secondary signal detection was performed by fitting the primary SNP as a fixed effect as implemented in FaST-LMM and using the same SNP set for the relationship matrix.

The decision to apply linear mixed models rather than principal-component analysis for population stratification was informed by a comparison of logistic regression with principal components as covariates against linear mixed models, including different strategies for computing the kinship (or similarity) matrix. In this analysis, we concluded that linear mixed models, when using a kinship matrix, outperformed logistic regression with principal components as covariates, as assessed by the genomic inflation factor in all SNPs (data not shown). We also noticed that including all SNPs when computing the kinship matrix resulted in further loss of power, particularly matrices calculated including $H L A-B^{\star} 27$-tagging SNPs, which are a good proxy for phenotype status in a case-control study such as ours. Thus, including SNPs tagging $H L A-B^{*} 27$ in the kinship matrix would have the effect of controlling for affected status, reducing statistical power in a case-control study.

Transformation of SNP effects from the linear 0-1 scale to the liability scale is described in the Supplementary Note. Association plots were generated using LocusZoom, with recombination rates estimated from the HapMap CEU panel (Utah residents of Northern and Western European ancestry) and pairwise LD $r^{2}$ values estimated from the set of control samples ${ }^{65}$.

Low-frequency SNP association analysis. A collection of 7,447 cases and 11,479 controls were selected for low-frequency SNP association analysis. These samples were of UK European origin according to principal-component analysis (Supplementary Fig. 11) and recruitment center informaiton. Association analysis was performed with Fisher's exact test and with logistic regression conditioning on common variant association within the locus.

Imputation. We imputed genotypes in candidate regions for the European and Asian cohorts using the EUR and ASN reference panels, respectively, from the 1000 Genomes Project (Phase 1, 2010-2011 data freeze) ${ }^{66}$. Genotype data were phased with $\mathrm{MACH}$, and genotypes were imputed with Minimac ${ }^{67}$. SNPs with low imputation quality $\left(r^{2}<0.5\right)$ were excluded. Association analysis in imputed genotypes was assessed with probabilistic genotypes, correcting for population stratification with the first five principal components as covariates, using logistic regression as implemented in mach2dat.

$\boldsymbol{H} \boldsymbol{L} \boldsymbol{A}-\boldsymbol{B}^{\star} \mathbf{2 7}$-tagging SNP. Imputed SNPs in the MHC region were tested for association and for tagging of $H L A-B^{\star} 27$. Performance as a tagging SNP was assessed with a cohort of samples previously genotyped at this locus (Supplementary Table 6b), including 754 controls from the 1958 Birth Cohort population $^{68}, 542$ ankylosing spondylitis cases from the UK and Australia and 104 ankylosing spondylitis cases and 5 controls from China. SNP rs 116488202 was used to tag $H L A-B^{\star} 27$ in all analyses.

Overlap with other autoimmune diseases. For all genome-wide significant and suggestive loci for ankylosing spondylitis susceptibility, we searched in a chromosomal window of $0.5 \mathrm{cM}$ around the lead SNP for associations with other autoimmune diseases in the National Human Genome Research Institute (NHGRI) GWAS catalog (accessed 5 June 2012). We then computed the LD between the ankylosing spondylitis-associated SNP and the reported SNP using phased data from the 1000 Genomes Project. If the ankylosing spondylitis-associated SNP was not found, we searched for the next most significant SNP in the locus. When a pair of SNPs was in $\mathrm{LD}$ (either $r^{2}>0.40$ or $D^{\prime}>0.40$ ), a connection between the two diseases was noted. Positively correlated alleles were then compared for their risk or protective effect on the two diseases to determine whether the directionalities of effect were concordant or discordant. LD was computed with Haploxt.

$H L A-B{ }^{\star} 27$ experiment-wide interactions. Testing for interaction between $H L A-B^{\star} 27$-tagging SNP rs116488202 and all other non-MHC SNPs was performed in samples of European ancestry by logistic regression fitting a dominant term for the $H L A-B^{\star} 27$-tagging SNP and an additive term for each 
test SNP, including a multiplicative interaction term, and five principal components for ancestry correction:

$$
y=\beta_{0}+\beta_{1} x_{1}+\beta_{2} x_{2}+\beta_{12} x_{1} \times x_{2}+\sum_{i=1}^{5} \beta_{\mathrm{PC}_{i}} \mathrm{PC}_{i}
$$

where $y$ is the $\log$ odds of disease, $\beta_{\mathrm{o}}$ is the intercept, $x_{1}$ is a SNP variable reflecting an $H L A-B^{\star} 27$ dominant effect (0 or 1$), x_{2}$ is a SNP variable coded to reflect an underlying additive effect $(0,1$ or 2$), \mathrm{PC}_{i}$ codes for the projection of samples onto the $i$ th principal component and $\beta$ terms are regression coefficients estimated from the data.

$\mathrm{CD4}^{+}$lymphocyte counts in ankylosing spondylitis cases and controls. Peripheral blood was obtained from 20 individuals with active ankylosing spondylitis (erythrocyte sedimentation rate of $>25 \mathrm{~mm} / \mathrm{h}$ and CRP concentration of $>10 \mathrm{mg} / \mathrm{l})$ who were naive for TNF inhibitor and 20 age-matched healthy controls. Peripheral blood mononuclear cells (PBMCs) were extracted using standard density gradient centrifugation over Ficoll-Paque Plus (GE Healthcare). Extracted PBMCs were frozen in FBS with 10\% DMSO until needed. Frozen PBMCs were thawed into RPMI supplemented with 20\% FBS and were washed once in RPMI supplemented with 10\% FBS. Cells were rested in RPMI supplemented with $10 \% \mathrm{FBS}$ for approximately $1 \mathrm{~h}$ at $37^{\circ} \mathrm{C}$ at $5 \% \mathrm{CO}_{2}$ before further use. Cells were stained with CD3 ECD (UCHT1, Beckman Coulter) and CD4 Pacific Blue (13B8.2, Beckman Coulter). Antibodies were used at final dilutions of 1:100 of antibody stocks. Dead cells were excluded using a Live/Dead Fixable Dead Cell Stain kit (Invitrogen). Cells were acquired on a Gallios flow cytometer (Beckman Coulter), and staining was analyzed using Kaluza software (Beckman Coulter).

IL-6R measurements in serum. Serum was collected from ankylosing spondylitis cases and controls of European ancestry attending the Princess Alexandra
Hospital Brisbane Ankylosing Spondylitis Specialist Clinic who were either homozygous for the $\mathrm{T}$ or $\mathrm{C}$ allele of rs4129267. Serum concentrations of IL-6R were measured using an IL-6R Quantikine ELISA (R\&D Systems), and optical density (OD) was determined on a Synergy 2 Microplate reader (BioTek). Serum was diluted 1:100, and ELISAs were performed according to the manufacturer's instructions. All data shown in graphs represent mean \pm s.e.m., and differences between groups were analyzed using non-parametric one-way ANOVA; Kruskal-Wallis test with Dunn's multiple-comparison post-hoc test in GraphPad Prism 5 software. Statistical significance was accepted at a significance level of $P<0.05$.

60. van der Linden, S., Valkenburg, H.A. \& Cats, A. Evaluation of diagnostic criteria for ankylosing spondylitis. A proposal for modification of the New York criteria. Arthritis Rheum. 27, 361-368 (1984).

61. Shah, T.S. et al. optiCall: a robust genotype-calling algorithm for rare, low-frequency and common variants. Bioinformatics 28, 1598-1603 (2012).

62. Fraley, C. \& Raftery, A.E. Model-based clustering, discriminant analysis, and density estimation. J. Am. Stat. Assoc. 97, 611-631 (2002).

63. Purcell, S. et al. PLINK: a tool set for whole-genome association and populationbased linkage analyses. Am. J. Hum. Genet. 81, 559-575 (2007)

64. Lippert, C. et al. FaST linear mixed models for genome-wide association studies. Nat. Methods 8, 833-835 (2011).

65. Pruim, R.J. et al. LocusZoom: regional visualization of genome-wide association scan results. Bioinformatics 26, 2336-2337 (2010).

66. 1000 Genomes Project Consortium.. A map of human genome variation from population-scale sequencing. Nature 467, 1061-1073 (2010)

67. Li, Y., Willer, C.J., Ding, J., Scheet, P. \& Abecasis, G.R. MaCH: using sequence and genotype data to estimate haplotypes and unobserved genotypes. Genet. Epidemiol. 34, 816-834 (2010).

68. Wellcome Trust Case Control Consortium. Genomewide association study of 14,000 cases of seven common diseases and 3000 controls. Nature 447, 661-678 (2007). 